\title{
PATHOLOGICAL COMPLETE RESPONSE IN 2,141 PATIENTS SUBMITTED TO NEOADJUVANT CHEMOTHERAPY IN A BREAST CANCER REFERENCE CENTER
}

Mayane Freitas Desiderio Silva ${ }^{1}$, Marcela Bonalumi Santos ${ }^{1}$, André Mattar ${ }^{1}$, Jorge Yoshinori Shida ${ }^{1}$, Luiz Henrique Gebrim ${ }^{1}$

${ }^{1}$ Hospital Pérola Byington - São Paulo (SP), Brazil.

Introduction: The pathologic complete response (pCR) definition after neoadjuvant chemotherapy (NAC) for breast cancer is better defined as the absence of residual invasive cancer, although it allows the presence of ductal carcinoma in situ (DCIS). In the past, the presence of positive axillary lymph node was allowed; nowadays studies have shown that any positive lymph node should be not considered as pCR. In Brazil, the proportion of advanced cases varies between $30 \%$ to $55 \%$ of patients treated by the public health system (SUS). NAC has been recommended more frequently, especially for triple negative tumors and overexpressed Her 2 tumors because you can possibly change the adjuvant treatment, and it is an excellent predictor of prognosis. There is little data on $\mathrm{PCR}$ in the therapeutic regimens used in SUS. Objectives: Evaluate data on pathologic complete response in patients subjected to neoadjuvant chemotherapy in SUS schemes. Methods: We performed a retrospective study in Pérola Byington Hospital official database from January 2011 to December 2018 and 2,141 patients that underwent neoadjuvant chemotherapy (NAC) were included. Chemotherapy regimens varied during this period according to institutional protocols and availability of new drugs. The pCR was considered after a histopathological study of the surgical specimen in four molecular subtypes (luminal A and B, triple negative and HER -2 enriched). Results: We included 494 patients in Stage IIB and 1,645 patients in Stage III, most of them were luminal (1,077/50.3\%), followed by triple negative (766/35,8\%) and Her2 (298/13,9\%). The pCR rate varied across the subtypes: luminal 78 (7.2\%), triple negative 163 (21.3\%) and Her2 69 (23.1\%). Conclusions: NAC is an important treatment for breast cancer and is gradually obtainingmore indications. Most of the indications for NAC are: To allow surgical approaches (advanced cases); To allow / increase rate of conservative surgery; To allow conservative approach to the axila and recently select some cases for specific treatment (adjuvant TDM1 and capecitabine). The pCR rate varies through the studies: Cortazar et al. found 16\% in Luminal; 33\% in triple negative; 50\% in Her2 when trastuzumab was used and 30,2\% when it was not used. When platin is used for triple negative it is possible to achieve up to $50 \%$ of pCR. When double blockage for Her 2 is used we can expect between 50\%-70\% in pCR. In our data most of our patients were stage III and we only had access to neoadjuvant trastuzumab after 2016; this directly impacted our results. The NAC protocols varied during this period and nowadays we have included platin compounds for triple negative. 